\title{
Micobatteriological control of diagnostic instrumentation
}

\author{
Valeria Olivo, Giulia Santoro, Gabriella Corsi, Francesco Russo, Elena Mosca, Riccardo Smeraglia \\ UOC Microbiologia e Virologia - AORN.V. Monaldi, Napoli
}

Key words: M. gordonae, bronchoscopies, lavaendoscopes

Controllo micobatteriologico di strumentazioni diagnostiche

\section{SUMMARY}

Introduction: In addition to sterility testings for common bacteria, from 2006 on we have introduced in our laboratory sterility tests for mycobacteria on surgical instruments such as bronchoscope and lava-endoscope fluid used for their sterilization as well.

Methods: Bronchology and Pneumology SUN departments, doing bronchoscopies, regularly send to our laboratory bronchoscopes washing fluids. Bronchology department also sends liquids used in lavaendoscopes. Our standard protocol consists of liquids inoculation directly on Lowenstein - Jensen (LJ) solid ground and on Middlebrook 7H9 liquid medium into Mgit test tubes, both produced by Becton Dickinson, within 42 days $(I, 5,6)$.

Results: When growth of acid-alcohol resistant bacilli was detected, they were identified through probes or Accuprobe bioMérieux InnoLIPA Mycobacteria by Innogenetics. All positivite results were related to M. Gordonae. As for the liquids examined, we found one positivite result in liquid medium in 2006 and none in 2007. On the contrary, in 2008 and the first 6 months of 2009, we have identified the following positive results: 2008 - $2009(6 \mathrm{~m})$ LJ pos TOT 20 C. Lavaendoscope 8V. 12 FBS We linked the positive findings on equipment with positivity for M. gordonae observed in patients undergoing bronchoscopy.

Conclusions: Data analysis indicates within the period 2008-2009 we observed a recurring positivity for M. gordonae on equipment and therefore in some patients. On a careful evaluation, they showed no signs of clinical infection, also considering that this mycobacteria is usually a saprophyte $(2,3,4)$. However, the protocol we adopted allowed us to recognize equipment contamination at birth and to urge fellow users to review sterilization protocols.

\section{INTRODUZIONE}

Dal 2006 abbiamo introdotto, nel nostro laboratorio, accanto al test di sterilità per germi comuni anche la prova di sterilità per Micobatteri su strumentazioni chirurgiche quali i broncoscopi e sui liquidi dei lavaendoscopi utilizzati per la sterilizzazione degli stessi.

\section{METODI}

I due reparti di Broncologia e Pneumologia SUN, che praticano broncoscopie, inviano abitualmente a questo Laboratorio i liquidi di lavaggio dei broncoscopi.

La Broncologia invia anche i liquidi utilizzati nei lavaendoscopi. Il nostro protocollo standard provvede l'inoculazione dei liquidi direttamente su terreno solido Lowenstein- Jensen e terreno liquido Middlebrook $7 \mathrm{H} 9$ in provette Mgit entrambi della Becton Dickinson per un periodo di 42 gg. $(1,5,6)$.

\section{RISULTATI}

Quando riscontrati, una produzione di bacilli acido alcool resistenti,vengono identificati da sonde Accuprobe bioMérieux o Inno-LiPA Mycobacteria della Innogenetics.

I campioni positivi hanno evidenziato la presenza di Mycobacterium gordonae.

Relativamente ai liquidi esaminati, abbiamo riscontrato una sola positività in medium liquido nel 2006 e nessuna nel 2007.

Nel periodo 2008-primi 6 mesi 2009 abbiamo invece riscontrato le seguenti positività (Tabella 1):

Tabella I.Distribuzione dei campioni nel periodo 2008-primi 6 mesi 2009

\begin{tabular}{lcccc}
\hline Origine dei campioni & LJ & LJ & Mgit & Mgit \\
& positivo & negativo & positivo & negativo \\
\hline Lavaendoscopi & $\mathbf{2 0}$ & $\mathbf{3}$ & $\mathbf{2 2}$ & $\mathbf{3}$ \\
\hline V.FBS (Videofibroendoscopio) & $\mathbf{8}$ & $\mathbf{0}$ & $\mathbf{8}$ & $\mathbf{0}$ \\
\hline LJ (Lowenstein - Jensen) & $\mathbf{1 2}$ & $\mathbf{3}$ & $\mathbf{1 4}$ & $\mathbf{3}$ \\
\hline
\end{tabular}

Abbiamo correlato le positività riscontrate sulle apparecchiature con le positività per $M$. Gordonae osservate nei pazienti sottoposti a broncoscopia. Tali dati sono riassunti in Tabella 2
Tabella 2. Correlazione tra positività riscontrata sugli strumenti e quella del paziente

\begin{tabular}{lccc}
\hline & $\begin{array}{c}\text { Attrezzature } \\
\text { positive }\end{array}$ & $\begin{array}{c}\text { Paziente } \\
\text { positivo }\end{array}$ & $\begin{array}{c}\text { Totale } \\
\text { attrezzature }\end{array}$ \\
\hline 2006 & I & $\mathbf{5}$ & I43 \\
\hline 2007 & $\mathbf{0}$ & I & I25 \\
\hline 2008 & $\mathbf{1 3}$ & $\mathbf{8}$ & $\mathbf{1 9 8}$ \\
\hline $2009(6$ mesi $)$ & $\mathbf{7}$ & $\mathbf{6}$ & $\mathbf{9 1}$ \\
\hline
\end{tabular}

\section{CONCLUSIONI}

L'analisi dei dati ci indica che nel periodo 2008-2009 abbiamo osservato una ricorrente positività per M.gordonae riscontrata sulle attrezzature e di conseguenza in taluni pazienti. Questi, ad una valutazione attenta, non hanno mostrato segni clinici di infezione anche tenuto conto che il micobatterio di cui discutiamo è, in genere, un saprofita $(2,3,4)$.

Tuttavia il protocollo da noi adottato ci ha consentito di cogliere sul nascere il problema della contaminazione delle attrezzature e di sollecitare i colleghi utilizzatori delle stesse ad una revisione dei protocolli di sterilizzazione.

\section{BIBLIOGRAFIA}

1. Bar W, Bar GM, Naumann A, Rusch-Gerdes S-Contamination of bronchoscopes with Mycobacterium tuberculosis and successful sterilization by low-temperature hydrogen peroxide plasma sterilization-Am J Infect Control 2001;29(5):306-11.

2. Daddi G, Pasargiklian M, Allegra M, Mancini P: Trattato di pneumologia , 1998 - vol III, 3 ed Piccin: 427-430.

3. Peduzzi R, Michelini V, Pagano E. Tubercolosi e micobatteriosi atipiche.Tribuna Medica Ticinese 1998;63(1):5-14.

4. Vassil GS: Opportunistic infections , 2003 - ed Humana Press: 81-114

5. Wenzel R, Edmond M. Tubercolosis infection after bronchoscopy. JAMA 1997; 278:1111.

6. World Health Organization. International Standard for Tuberculosis Care - Seventh Draft. Geneve, 2005.

\section{Corresponding author: Giulia Santoro}

UOC Microbiologia e Virologia - AORN "V. Monaldi"

80I3I Napoli, Italy - Via Leonardo Bianchi - Tel 08I 706253।

E-mail: giulia.santoro@ospedalemonaldi.it 